\title{
Non linear predictions from linear theories in models with Dark Energy
}

\author{
R. Mainini, A.V. Macciò \& S.A. Bonometto \\ Physics Department G. Occhialini, Università degli Studi di Milano-Bicocca, \\ Piazza della Scienza 3, I20126 Milano (Italy) \\ I.N.F.N., Via Celoria 16, I20133 Milano (Italy)
}

\begin{abstract}
We study the cluster mass function and its evolution in different models with Dark Energy arising from a self-interacting scalar field, with Ratra-Peebles and SUGRA potentials. Computations are based on a Press \& Schechter approximation. The mass functions we obtain are compared with results holding for open models or models with Dark Energy due to a cosmological constant. Evolution results, in some Dark Energy models, closely approach open models.
\end{abstract}

Key words: methods: analytical, numerical - galaxies: clusters - cosmology: theory - dark energy

\section{Introduction}

One of the main puzzles in modern cosmology is the nature of Dark Energy (DE), whose presence seems to be required by SNIa data (see, e.g., Perlmutter et al 1999, Riess et al 1998). A joint analysis of CMB and LSS observations (see, e.g., Percival et al. 2002, Efstathiou et al 2002) also favor a flat Universe with a matter density parameter $\Omega_{m} \simeq 0.3$, mostly due to CDM and with a minor contribution of baryons $\left(\Omega_{b} h^{2} \simeq 0.02 ; h\right.$ is the Hubble constant in units of $100 \mathrm{~km} / \mathrm{s} / \mathrm{Mpc}$; in this paper we shall take $h=0.7$ and, unless differently specified, $\Omega_{m}=0.3$, anywhere). The residual energy content of the world, in the present epoch, should not be observable in the number-of-particle representation.

1 E-mails: mainini@mib.infn.it, maccio@mib.infn.it, bonometto@mib.infn.it 
One of the most appealing possibilities is that such dark component arises from a self-interacting scalar field. With in the wide set of interaction potential suggested, a particular relevance is kept by Ratra-Peebles (1988, RP hereafter; see also Wetterich 1995) and SUGRA (Brax \& Martin 1999, 2000) expressions:

$$
V(\phi)=\Lambda^{4+\alpha} / \phi^{\alpha}, \quad V(\phi)=\left(\Lambda^{4+\alpha} / \phi^{\alpha}\right) \exp \left(\kappa \phi^{2} / 2\right)
$$

Here $\Lambda$ is an energy scale, currently set in the range $10^{2}-10^{10} \mathrm{GeV}$, relevant for fundamental interaction physics; potentials depend also on the exponent $\alpha$; fixing $\Lambda$ and $\alpha$, the DE density parameter $\Omega_{D E}$ is automatically fixed; in this work we preferred to use as free parameters $\Lambda$ and $\Omega_{D E}$; in SUGRA potentials, $\kappa=8 \pi G(G$ : gravitational constant).

In this work we try to determine some effects on galaxy clusters and their evolution, caused by replacing a simple cosmological constant with DE due to a scalar field self-interacting according to the potentials (1).

The technique used to study non-linear evolution is the Press \& Schechter (PS, Press \& Schechter 1974) approach. It is based on the spherical collapse model, that Gunn \& Gott (1972), Gott \& Rees (1975), Peebles (1980) debated within the frame of pure CDM models, and Lahav et al (1991), Eke et al (1996), Brian \& Norman (1998) and others generalized to the case of $\Lambda$ CDM. In spite of its approximation, such model, inserted in PS formulation, has been found to approach simulation results (see, e.g., Lacey \& Cole 1993, 1994). Recent improvements of the method (Sheth \& Tormen 1999, 2002, Sheth, Mo \& Tormen, 2001, see also Jenkins et al 2001), allowing a better approximation, involve some more parameters and their use seems unnecessary when aiming just to compare different cosmological models.

\section{From linear theory to non linear predictions}

In order to apply the PS technique we first need to determine the amplitude $\delta_{c}$, in the linear theory, of a spherical fluctuation that would achieve full recollapse at a given redshift $z_{c o l}$. Real fluctuations, after achieving maximum expansion, turn around and begin to recontract. However, contraction requires that potential energy, turned into internal kinetic energy, is radiated soon. If radiation is negligible, contraction is delayed and, at $z_{c o l}$, virial equilibrium is attained. In a standard CDM model, $\delta_{c}$ is constant and holds $\sim 1.68$ (see, e.g., Coles \& Lucchin 1995). In RP or SUGRA models, $\delta_{c}$ depends upon the cosmological parameters and the redshift $z_{c o l}$. In Fig.s 1 and 2 we report such dependence. As a by-product of such computation, we obtain the density contrast $\left(\Delta_{c}\right)$ of a spherical fluctuation when it is fully virialized. In Fig. 3 we report the dependence of $\Delta_{c}$ on $\Omega_{m}$ at $z=0$, for a number of DE models. 


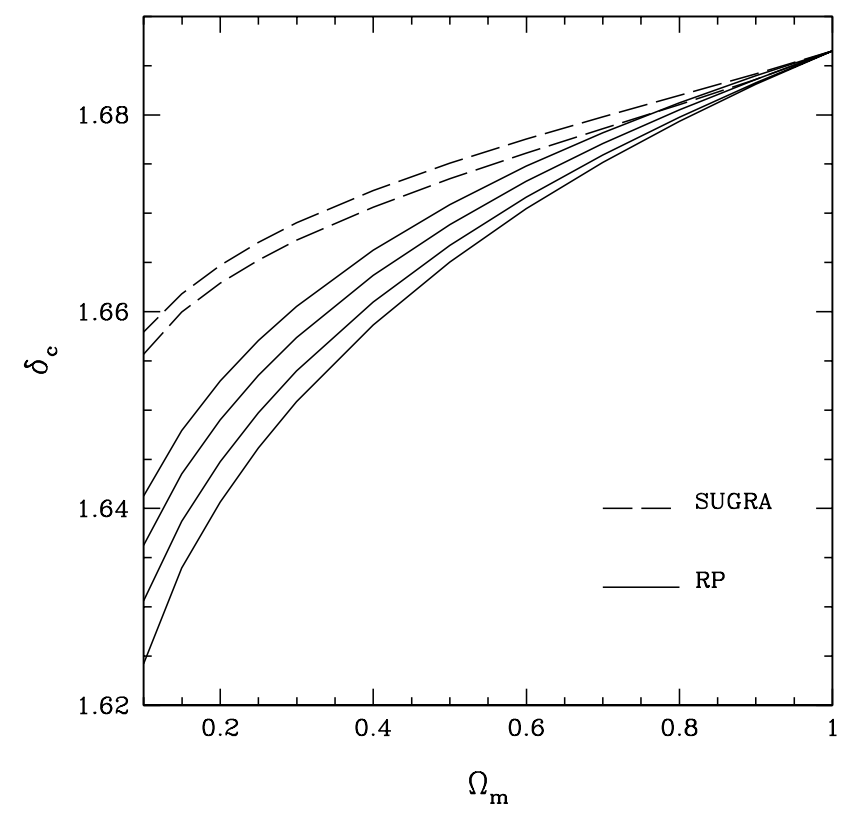

Fig. 1. The dependence of $\delta_{c}$ on the matter density parameter $\Omega_{m}$ is shown, for 4 $\mathrm{RP}\left(\Lambda / \mathrm{GeV}=10^{2}, 10^{4}, 10^{6}\right.$ and $\left.10^{8}\right)$ and 2 SUGRA models $\left(\Lambda / \mathrm{GeV}=10^{2}\right.$ and $\left.10^{8}\right) . \Lambda$ values increase from top to bottom curves.

Then, in Fig. 4, we report how $\Delta_{c}$ depends on $z$ for some DE models, keeping $\Omega_{m}=0.3$ and $h=0.7$.

The value of $\delta_{c}$ is to be used in the expression

$$
f(\nu) \nu d \log \nu=\frac{M}{\bar{\rho}_{m}} n_{c}(M) M d \log M
$$

(here $\bar{\rho}_{m}$ is background matter density, the bias factor $\nu=\delta_{c} / \sigma_{M}, \sigma_{M}$ being the rms density fluctuation on the length scale corresponding to the mass $M$ ), yielding the differential mass function $n_{c}(M)$ once the distribution on bias is given. Here, as usual, we assume a Gaussian $f(\nu)$. Eq. (2) shall then be integrated, to obtain $n_{c}(>M)$. Let us outline that, in order to compute $\sigma_{M}$, we need to know the transfered spectrum $P(k)$; let us also recall the relation $k=2 \pi(4 \pi \bar{\rho} / 3 M)^{1 / 3}$ between wavenumber and mass.

The first finding of this analysis is that the difference between the mass functions for RP or SUGRA models and the mass function of a $\Lambda$ CDM model, at $z=0$, is smaller than the difference due to a shift by 0.05 of the primeval spectral index. The intrinsic noise of data is then a serious obstacle to any attempt to determine the nature of DE using cumulative mass functions at $z=0$.

On the contrary, we found a clear imprint of the nature of DE in the evolution 


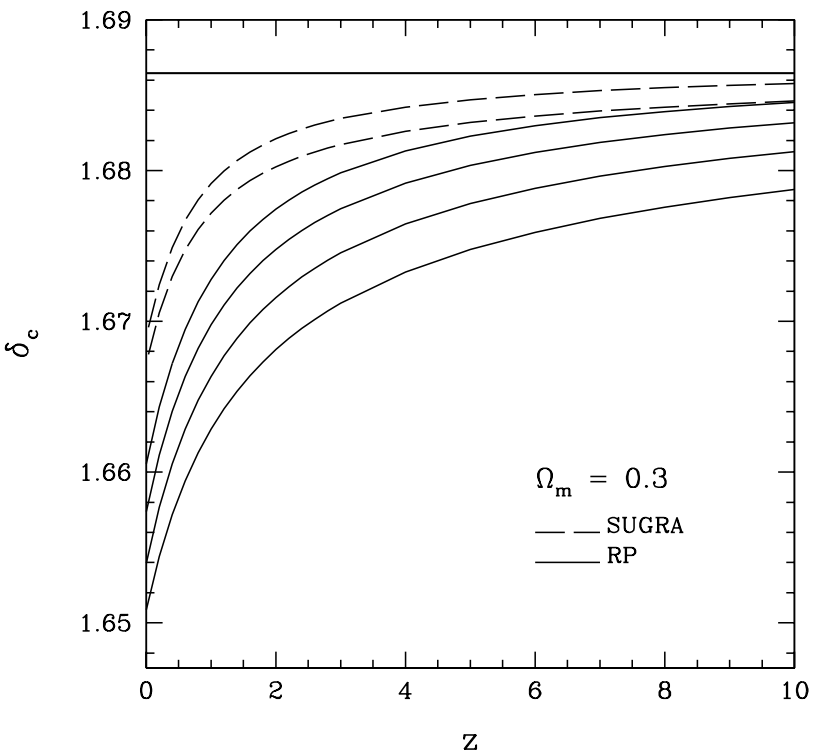

Fig. 2. The dependence of $\delta_{c}$ on the redshift $z$ is shown for $\Omega_{m}=0.3$ and $h=0.7$. $\Lambda$ values and curve setting are the same as in Fig. 1 . 


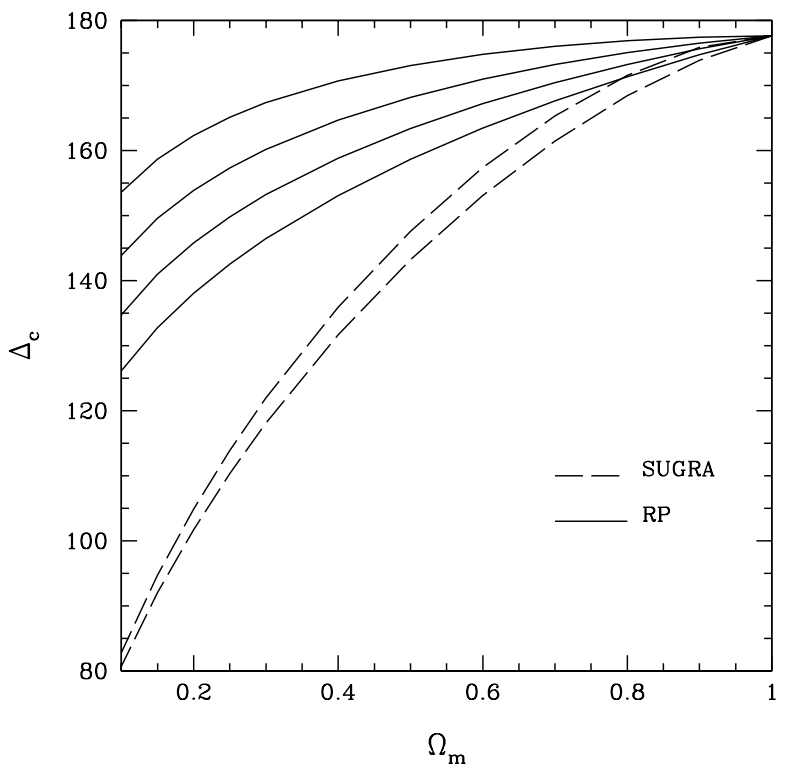

Fig. 3. The density contrast $\Delta_{c}$ of a virialized sphere is shown for $4 \mathrm{RP}$ and 2 SUGRA models as a function of $\Omega_{m}$, for $h=0.7$, at $z=0 . \Lambda$ values are the same as in Fig. 1, but here they decrease from top to bottom curves.

of the number $N$ of clusters (above a suitable mass $M$, in a box of side $s$ ). In Fig. 5, we give $N$ (for $M>6.9 \cdot 10^{14} h^{-1} M_{\odot}$ and $s=100 h^{-1} \mathrm{Mpc}$ ) as a function of redshift, normalized to an identical number of clusters at $z=0$, for all models $(N(z=0)=0.13)$. In Fig. 6 we give the ratio between the number of clusters expected in various models and the number expected in an open CDM model with the same value of $\Omega_{m}$. The mass here is selected so to correspond to a cluster with Abell radius in a standard CDM model. A similar plot, for a slightly smaller mass, was given by Bahcall, Fan \& Cen (1997), for standard CDM, $\Lambda$ CDM and OCDM only (for a recent analysis of the constraints that cluster number counts set to the cosmological model, see, e.g., Holder, Haiman \& Mohr, 2001).

\section{Discussion}

The above mass functions and evolution are calculated using a transfer function obtained from a generalization of the public program CMBFAST to cosmologies with DE given by the potentials (1). Initial conditions were set according to the tracker solution in radiation dominated era (Steinhardt, Wang \& Zlatev 1999; Zlatev, Wang, \& Steinhardt 1999, Brax, Martin \& Riazuelo 2000). DE fluctuations, taken into account in primeval fluctuation evolution, are damped soon after a scale enters the horizon and, therefore, are not important in further fluctuation evolution. Accordingly, in our problem, their 


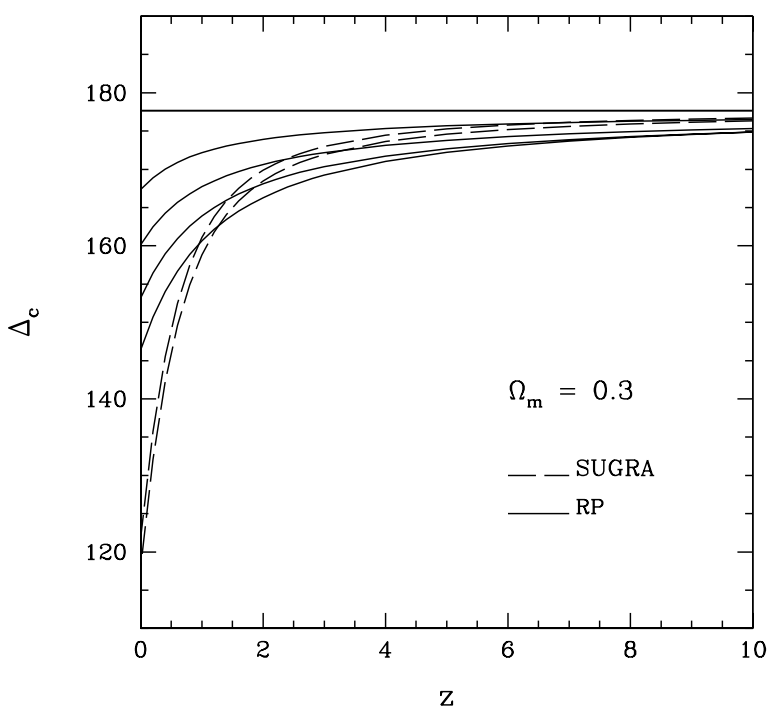

Fig. 4. The density contrast $\Delta_{c}$ of a virialized sphere is shown for $4 \mathrm{RP}$ and 2 SUGRA models as a function of $z$. For the sake of comparison, the constant value of $\Delta_{c}$ in standard CDM is also reported. $\Lambda$ values and curve setting are the same as in Fig. 3. 
relevance amounts to causing some modification of the transfer function. (On the contrary, they cause major modifications of CMB anisotropy and polarization.)

Fig. 7 describes the pattern followed to evaluate $\delta_{c}$ and $\Delta_{c}$ for $z_{c o l}=0$; for $z_{c o l}>0$ the procedure is similar. It must be however outlined that Fig. 2 does not describe a time evolution, but is worked out considering different time evolutions of the kind described in Fig. 7. We obtain the linear growth factor by using a simplified program, suitable to treat fluctuation evolution after recombination, which includes the Friedman equation, the equation of motion of the scalar field and the Jeans' equation for the linear density fluctuation $\delta$ :

$$
\ddot{\delta}+2 \frac{\dot{a}}{a} \dot{\delta}-\frac{3}{2} \frac{H_{o} \Omega_{m}}{a^{3}} \delta=0
$$

(dots yield derivatives in respect to time, $H_{o}$ is the present value of the Hubble constant). Such system provides the lower curves in Fig. 7; for a flat model without DE (SCDM), the curve is a straight line; together with it, in the figure, we show the behavior for a RP model. Other models, with different DE contents, have a similar behavior. Such linear results can be multiplied by an arbitrary factor and, in the figure, they are normalized so that the initial fluctuation amplitude allows full recollapse, at $z_{c o l}$, of a spherically symmetric fluctuation. This is also to be done in actual calculations to evaluate $\delta_{c}$. The evolution of the contrasts $\Delta=\rho_{m} / \bar{\rho}_{m}$, between inner and average matter densities, is then shown by the upper curves. However, instead of reporting their diverging at $z_{c o l}$, we interrupt their growth when virialization density contrast is attained; the residual slight growth of $\Delta$ is due to cosmic expansion. Notice that the density contrasts $\Delta_{c}$, shown in Fig.s 3 and 4 , are $\Omega_{m} \Delta$.

More in detail, within such spherical lump, at an initial redshift $z_{i}$, the energy density exceeds average by a factor $1+\delta_{i}=\Delta_{i}$, tuned so cause full recollapse at an assigned redshift $z_{c o l}=1 / a_{c o l}-1$. The radius $R$ of the lump starts from $R_{i}$; its evolution is then computed by taking into account, besides of the varying energy density $\left(\rho_{m}\right)$ of the baryon and dark matter within $R$, also the energy density and pressure of $\mathrm{DE}\left(\rho_{D E}\right.$ and $p_{D E}$, respectively), within $R$ itself. In models involving just a cosmological constant, it is $-p_{D E}=\rho_{D E}=$ const., during the whole process (see, e.g., Lahav et al 1991). In the present case, instead, $p_{D E}, \rho_{D E}$ and their ratio vary in time, simply because of their overall evolution with $a$. At turn-around the radius will be $R_{t a}$. Afterwards, $R$ decreases and should vanish at $z_{c o l}$. The ratio between the energy density inside $R$ and average yields $\Delta$.

For the sake of example, in Fig. 8 we show $R(t)$ for two RP potentials $(\Lambda / \mathrm{GeV}=$ $10^{2}$ and $10^{8}$ ) and $\Lambda$ CDM. SUGRA potentials yield curves intermediate between $\mathrm{RP}$ and $\Lambda \mathrm{CDM}$ models and just slightly dependent on $\Lambda$. A careful inspection of the figures shows that, at variance form SCDM and $\Lambda$ CDM, RP models 


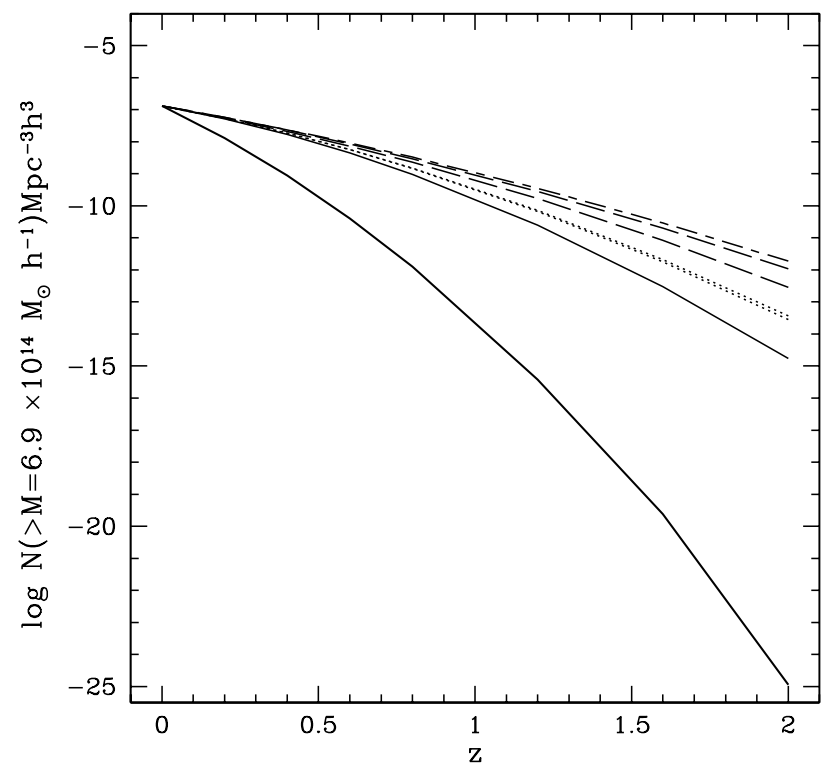

Fig. 5. The number density of clusters is shown for standard CDM (lowest curve), OCDM (long-short dashed upper curve), $\Lambda$ CDM (solid intermediate curve), 2 RP (long dashed curves) and 2 SUGRA (dotted curves) models. The values of $\Lambda / \mathrm{GeV}$ are $10^{2}, 10^{6}$ for RP and $10^{6}, 10^{10}$ for SUGRA, starting from the lower curves.

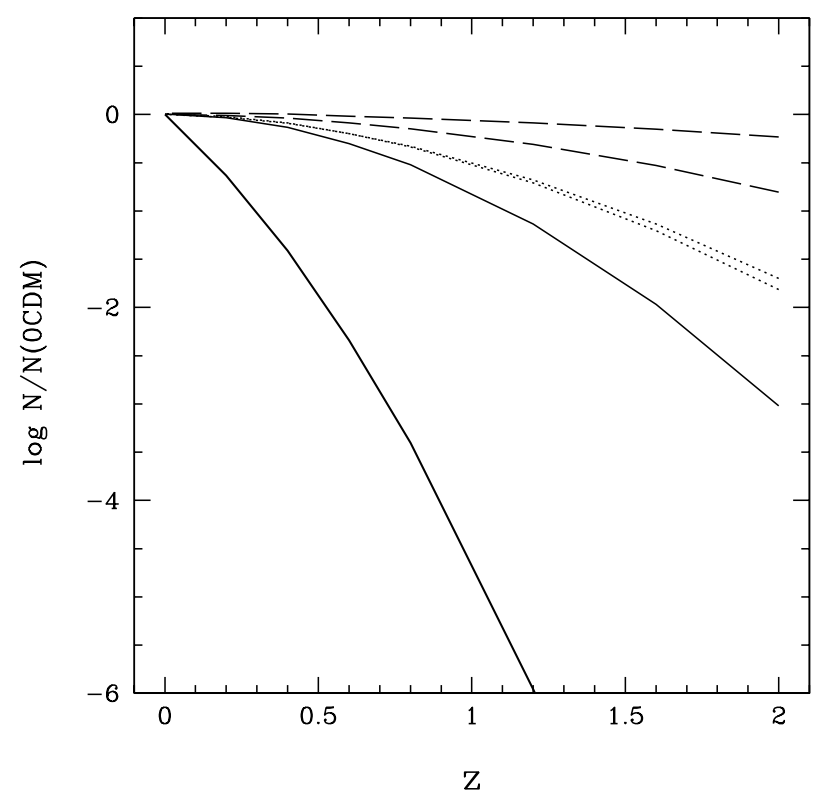

Fig. 6. The number densities of the previous plot normalized to the values in OCDM are shown. 


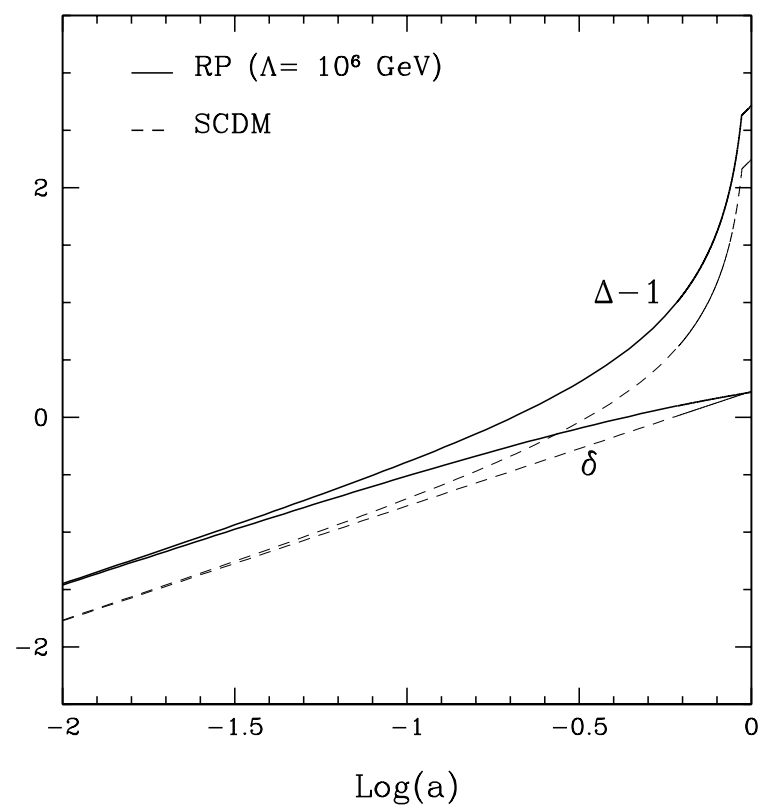

Fig. 7. Linear and non-linear evolution of density fluctuations. A models with DE due to a self-interacting scalar field is compared with SCDM. Similar plots can be given when full recollapse is expected when $a<1$. The density contrasts $\Delta_{c}$, shown in Fig.s 3 and 4, are $\Omega_{m} \Delta$.

have a slight asymmetry between expansion and recontraction, due to the evolution of DE density. In Fig. 8, the virialization radius $R_{v i r}$, from which $\Delta_{c}$ is computed, is also indicated. In the SCDM case, energy conservation yields $R_{v i r}=R_{t a} / 2$. In the presence of $\mathrm{DE}$, requiring virial equilibrium and energy conservation leads to the cubic equation

$$
x^{3}-\left[\frac{2+\eta\left(a_{t a}\right)}{2 \eta\left(a_{c o l}\right)}\right] x+\frac{1}{2 \eta\left(a_{c o l}\right)}=0 .
$$

Here $x=R_{v i r} / R_{t a}$ and

$$
\eta(a)=2 \frac{1-\Omega_{m}(a)}{\Omega_{m}(a)\left(1+\Delta_{i}\right)}\left(\frac{R_{t a}}{R_{i}}\right)^{3}\left(\frac{a_{i}}{a}\right)^{3}
$$

$\left(\Omega_{m}(a)\right.$ is the matter density parameter when the scale factor is $a$; accordingly, $\left.\Omega_{m}(1) \equiv \Omega_{m}\right)$. Solving such equation yields $x$ values slightly below 0.5 .

\section{Conclusions}

Observable effects of the nature of DE have been considered by various authors. For instance, Cooray \& Huterer (1999) discussed the relation between 


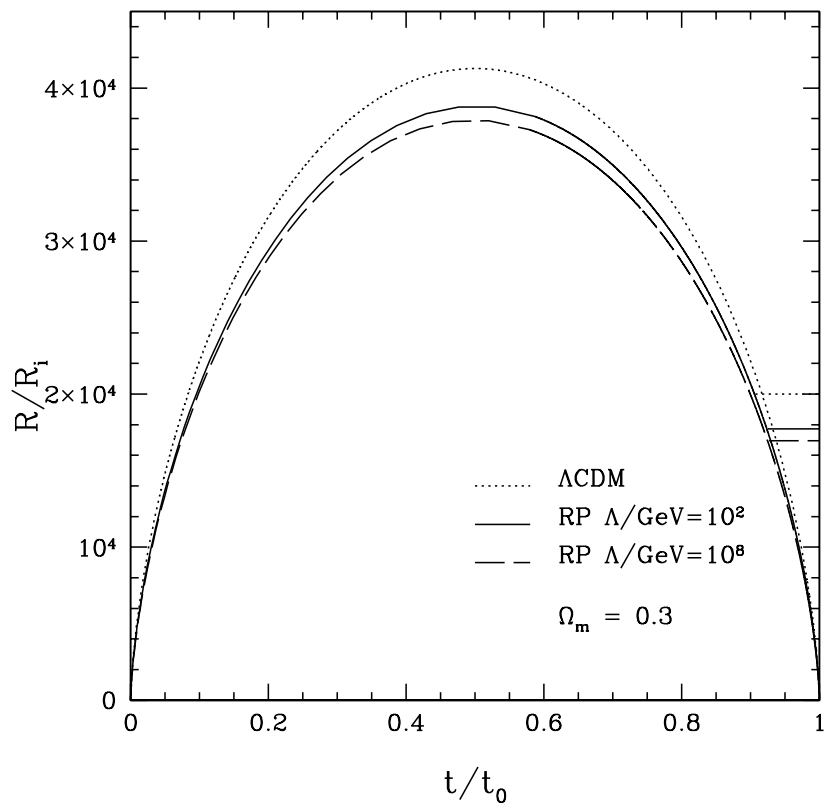

Fig. 8. Time dependence of the radius of a density fluctuation in various models; horizontal lines, at the right, indicate the $R_{v i r} / R_{i}$ attained at $t_{o}$. Similar plots can be given when full recollapse is expected at $t<t_{0}$. 


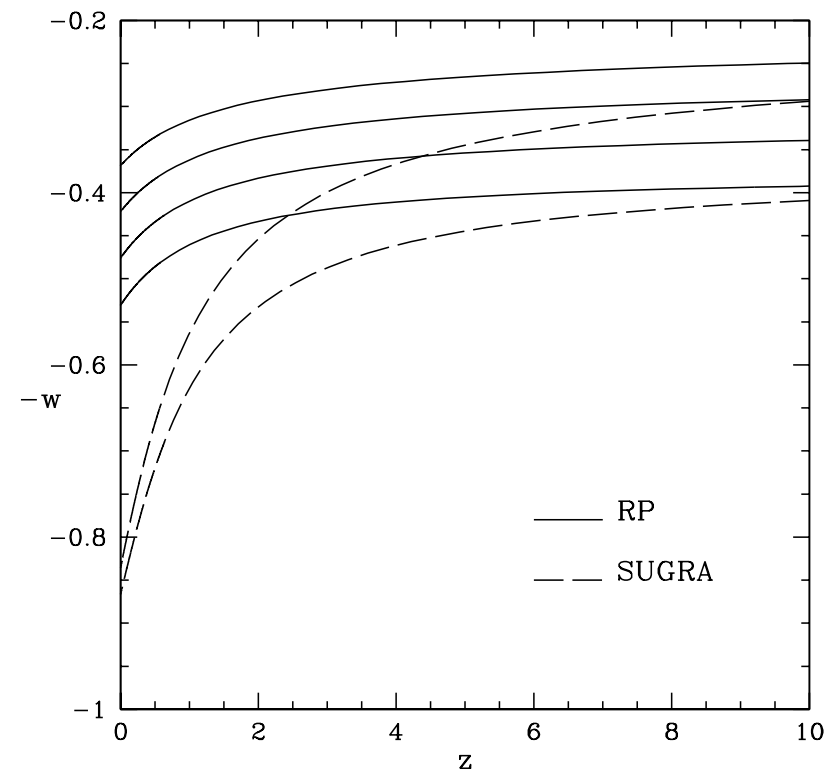

Fig. 9. Redshift dependence of $w$ for $4 \mathrm{RP}\left(\Lambda / \mathrm{GeV}=10^{2}, 10^{4}, 10^{6}\right.$ and $\left.10^{8}\right)$ and 2 SUGRA models $\left(\Lambda / \mathrm{GeV}=10^{2}\right.$ and $\left.10^{8}\right) . \Lambda$ values decrease from top to bottom curves.

DE nature and gravitational lensing. Previous work on the value of $\delta_{c}$ was made by Steinhardt, Wang \& Zlatev (1999), although explicit outputs were not given. More recently, Lokas (2002) considered the behavior of $\Delta_{c}$ and the mass function in DE models with constant $w=-p / \rho$. This approximated treatment has been pursued in a number of recent papers and eases computations. Let us however remind that the value of $w$, when dynamical DE is considered, varies significantly in the relevant period. In Fig. 9 we report its variations between $z=0$ and 10, when structures form. Notice that the rate of $w$ variation is highest in the most critical redshift interval, between $z=0$ and $1-2$. In RP models such variation is $\sim 20 \%$. In SUGRA models it is even greater, as $w$ passes from values $\sim 0.8$, at $z=0$, up to values $\sim 0.3-0.4$ in the above narrow $z$ interval. Taking constant $w$, therefore, is a dangerous approximation, whose reliability ought to be carefully inspected, in different problems.

The main results of this work are that: (i) the shape of the mass function of clusters, at $z=0$, is only mildly dependent on DE nature. On the contrary, (ii) the cluster evolution depends on the nature of DE in a significant way. More in detail, models with RP potentials closely approach the evolution expected in open CDM models. Only for $\Lambda$ values as low as $\sim 10^{2} \mathrm{GeV}$, the expected behavior in a RP model is appreciably different from an open CDM with the same $\Omega_{m}$. On the contrary, the evolution of SUGRA models is intermediate between open CDM and $\Lambda$ CDM models. 
Cluster data available within a few years were thought to be able to discriminate between open CDM and $\Lambda \mathrm{CDM}$, on the basis of the redshift dependence of cluster abundance. If independent data confirm that we live in a spatially flat world, finding an evolution closer to open CDM than to $\Lambda$ CDM will provide a precise information on the nature of $\mathrm{DE}$.

As a by-product of the analysis leading to these conclusions, we also found the dependence of the virialization density contrast $\Delta_{c}$ on $\Omega_{m}$, on the nature of $\mathrm{DE}$, and on redshift $z$. Such $\Delta_{c}$ is to be used in various applications, e.g. to build SO algorithms able to find clusters in n-body simulations of models with DE.

\section{References}

[1] Bahcall N., Fan X. \& Cen R., 1997, ApJ, 485L, 53B

[2] Brax, P. \& Martin, J., 1999, Phys.Lett., B468, 40

[3] Brax, P. \& Martin, J., 2000, Phys.Rev. D, 61, 103502

[4] Brax P., Martin J. \& Riazuelo A., 2000, Phys.Rev. D, 62, 103505

[5] Brian, G. \& Norman, M., 1998, ApJ, 495, 80

[6] Coles, P. \& Lucchin, F., 1995, Cosmology, John Wiley \& Sons.

[7] Cooray, A.R. \& Huterer, D., 1999, ApJ, 513L, 95C

[8] Efstathiou, G. et al., 2002, MNRAS, 330, 29

[9] Eke, V.R., Cole, S., \& Frenk, C.S., 1996, MNRAS, 282, 263

[10] Gott, R. \& Rees, M. 1975, A\&A, 45, 365G

[11] Holder, G., Haiman, Z. \& Mohr, J.J., 2001, ApJ, 560L, 111H

[12] Jenkins, A., Frenk C.S., White S.D.M., Colberg J.M., Cole S., Evrard A.E., Couchman H.M.P. \& Yoshida N., 2001, MNRAS, 321, 372

[13] Lahav, O., Lilje, P.R., Primack, J.R. \& Rees, M., 1991, MNRAS, 282, 263E

[14] Lacey, C.\& Cole, S., 1993, MNRAS, 262, 627

[15] Lacey, C.\& Cole, S., 1994, MNRAS, 271, 676

[16] Lokas, E.L., 2002, astro-ph/0112031

[17] Peebles P.J.E., 1980, The Large Scale Structure of the Universe, Princeton University Press, Princeton

[18] Percival W.J. et al. astro-ph/0206256 
[19] Perlmutter S. et al., 1999, ApJ, 517, 565

[20] Press W.H. \& Schechter P., 1974, ApJ, 187, 425

[21] Ratra B., Peebles P.J.E., 1988, Phys.Rev.D, 37, 3406

[22] Riess, A.G. et al., 1998, AJ, 116, 1009

[23] Sheth R.K., Mo H.J. \& Tormen G., 2001 MNRAS, 323 ,1

[24] Sheth R.K. \& Tormen G., 1999 MNRAS, 308, 119

[25] Sheth R.K. \& Tormen G., 2002 MNRAS 329, 61

[26] Steinhardt P.J., Zlatek, I. \& Wang L., 1999, Phys Rev D, 59, 123504

[27] Wetterich C., 1995 A\&A 301, 32

[28] Zlatek, I., Wang L. \& Steinhardt P.J., 1999, Phys Rev Lett, 82, 896 